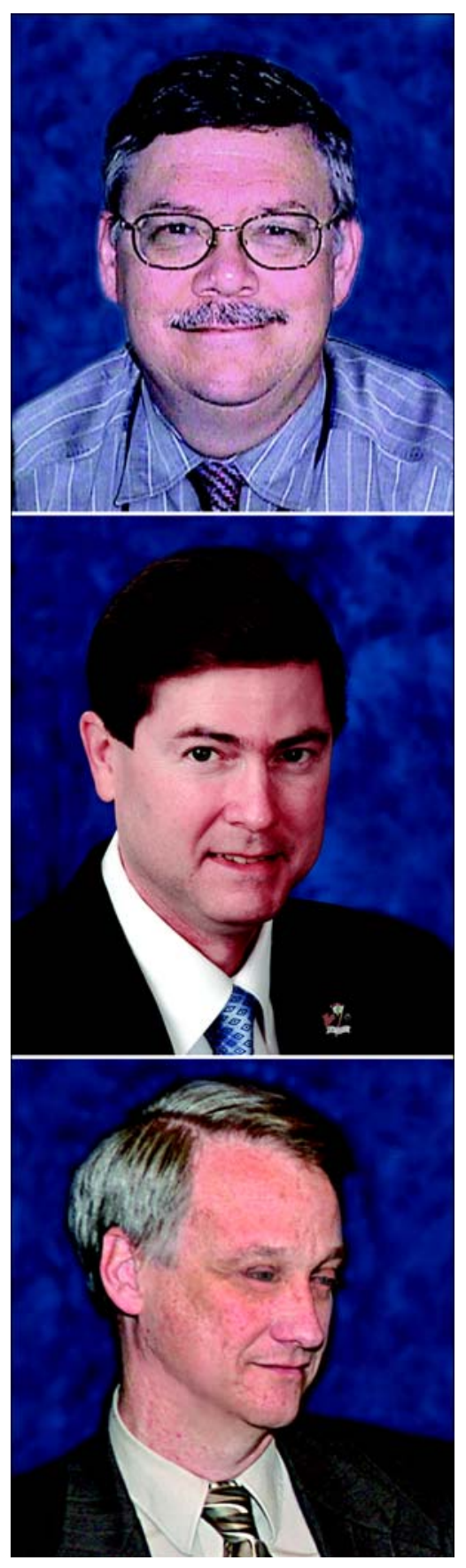

James C. Orcutt, MD, PhD

John C. Townsend, OD, FAAO

Ronald A. Schuchard, PhD

\section{Vision rehabilitation: Current research activities help- ing to meet needs of veterans with visual impairments}

According to the National Eye Institute of the National Institutes of Health, Americans are facing increasing rates of visual impairment and blindness from age-related diseases. More than 1 million Americans aged 40 and over are currently blind and an additional 2.4 million are visually impaired. These numbers are expected to double over the next 30 years as the baby-boomer generation ages.

Vision Problems in the U.S. (http://www.nei.nih.gov/eyedata/pdf/VPUS.pdf), a report on the prevalence of sight-threatening eye disease in Americans, addresses the leading causes of visual impairment and legal blindness, including-

- Diabetic retinopathy, a leading cause of visual impairment in the industrialized world among people aged 25 to 74 .

- Age-related macular degeneration, the most common cause of visual impairment in Americans 60 and older.

- Cataract, the leading cause of blindness in the world, although not a significant factor in the United States.

- Glaucoma, a chronic disease that often requires life-long treatment.

The articles in this special issue on vision address three general areas: screening for diabetic retinopathy using teleretinal imaging, basic research on treating and preventing vision loss, and management of patients with low vision or legal blindness.

In addition to an increased prevalence of vision loss, many older adults have concomitant hearing loss that results in a dual sensory impairment. Dual sensory impairment affects anywhere from 5 to 21 percent of older adults. Although much lower in prevalence than vision loss, this combined vision and hearing loss is associated with much greater functional limitations in the performance of activities of daily living (ADL). Visual and aural rehabilitation programs must concomitantly compensate for these changes and improve functional competence in everyday tasks.

The Veterans Health Administration (VHA) has been involved in the VHA Teleretinal Imaging Program, a project that uses telemedicine to screen veterans with diabetes for diabetic retinopathy. This project seeks to capture veterans with diabetes who are not being examined by optometrists and/or ophthalmologists for diabetic retinopathy. Retinal images of these veterans with diabetes will be obtained while they are seen in a primary care clinic setting. Afterward, these images will be transmitted to a reading site for reading by an optometrist or ophthalmologist. Pilot programs were started in Veterans Integrated Service Network (VISN) 1 and VISN 20. The success of these initial programs led the VHA to expand the VHA Teleretinal Imaging Program within the VHA Office of Care Coordination. 
In each of the 17 selected VISNs, the VHA will place six imaging cameras at various locations along with imagers and readers who have received standardized education at the Boston Department of Veterans Affairs (VA) Teleretinal Imaging Training Center. As of September 2006, more than 70 cameras have been installed in 13 VISNs. More than 10,000 veterans' retinal images have been taken and read by specially trained eye doctors, optometrists, and ophthalmologists. The VHA Teleretinal Imaging Program is anticipated to be fully implemented in early 2007 and to improve access for our diabetic patients.

When visual impairment and blindness occur despite preventive measures or following medical therapy and/or surgical intervention, one must determine the degree of the patient's visual impairment and provide vision rehabilitation services. The scanning laser ophthalmoscope has proven to be a unique testing instrument and is often the gold standard for performing macular perimetry and determining a patient's preferred retinal locus (PRL) characteristics. For example, while individuals without a visual impairment typically use the fovea to scan and fixate text, individuals with central scotomas or blind spots from macular degeneration scan text with a PRL, a peripheral retinal area that is used to scan and fixate objects. By using the scanning laser ophthalmoscope to determine an individual's retinal text map relative to the PRL, clinicians may be able to tailor rehabilitative strategies and improve the effectiveness of reading training.

The goal of restoring sight to the blind is moving from dream to possibility. One line of research that continues to evolve is the restoration of functional vision through implanted retinal prosthetic devices. Such prosthetic devices provide a neurotrophic effect that stimulates nerve growth factors in the retinas of patients with photoreceptor degeneration. For patients with no remaining functional vision, a retinal prosthetic will soon provide functional vision for everyday tasks by detecting light with a camera and stimulating the retina with an implanted chip. Any successful implant must be biocompatible and demonstrate long-term durability. One day, regeneration of essential retinal elements may be possible, as demonstrated by basic research on the migration and proliferation of retinal pigment epithelium that may eventually repair retinal damage. Hope exists that one day combined basic science research and prosthetic device development may restore functional vision to patients who are legally blind.

With the promise of new technologies, one should not forget that these novel clinical care and research strategies must ultimately benefit the patient. Therefore, these strategies require validated outcomes measures that have been fully tested and designed to evaluate the essential everyday functional abilities of patients with visual impairments and patients with profound vision loss. These measures should be used for acquiring evidence that new technologies and innovative rehabilitative interventions improve ADL performance and quality of life. In addition, one must keep in mind that the timing and directions provided when administering any outcomes assessment tool may influence the vision rehabilitation outcomes attained.

With the aging of the U.S. population and the rising prevalence of diseases and disorders that affect vision, many challenges face our nation regarding the prevention, evaluation, treatment, and rehabilitation of visual impairments. We hope that this special issue on vision will stimulate new ideas and encourage further vision rehabilitation research activities to better meet the needs of patients who are blind or visually impaired.

\section{John C. Townsend, OD, FAAO ${ }^{1}$ James C. Orcutt, MD, PhD; ${ }^{2 *}$ Ronald A. Schuchard, PhD $^{3}$}

${ }^{1}$ Optometry Service, Baltimore VA, Baltimore, MD; ${ }^{2}$ Department of Ophthalmology, VA Puget Sound Health Care System, Seattle, WA; ${ }^{3}$ Blind Rehabilitation Service, Rehabilitation Research and Development Center of Excellence for Aging Veterans with Vision Loss, Atlanta VA Medical Center, Decatur, GA

*Email: james.orcutt@med.va.gov

DOI: 10.1682/JRRD.2006.10.0126 